\title{
Organizational Safety Climate and Work Experience
}

\author{
Seth Ayim Gyekye \\ Psychology Department, Buckinghamshire New University, High Wycombe, Bucks, UK
}

Simo Salminen

Human Factors at Work, Finnish Institute of Occupational Health, Helsinki, Finland

\begin{abstract}
The study examined the relationships between work experience and (a) safety perceptions, (b) job satisfaction, (c) compliance with safety management policies and $(d)$ accident frequency. Participants were Ghanaian industrial workers $(\mathrm{N}=320)$. They were divided into 2 cohorts: experienced and inexperienced workers. Workplace safety perceptions were assessed with Hayes et al.'s 50-item work safety scale. MANOVA was used to test for differences of statistical significance. Posterior comparison with $\mathrm{t}$ test consistently revealed significant differences between experienced cohorts and their inexperienced counterparts. Experienced workers indicated the best perceptions on safety, expressed the highest level of job satisfaction, were the most compliant with safety procedures and recorded the lowest accident frequency. From a practical perspective, analysing differences in work experience in relation to safety perceptions could be useful for organizations as the workers' experience could indicate a need for special safety programmes for particular groups.
\end{abstract}

work experience safety perceptions job satisfaction compliance safety management

\section{INTRODUCTION}

Research on perception of workplace safety began in the early 1980s with Zohar's study [1], and has since received considerable attention in organizational and psychological literature. The perceptions about safety values, norms, beliefs, practices and principles shared by workers in their work environments have been technically termed safety climate [1, 2]. Zohar [1, 3], Cooper and Phillips [2], Prussia, Brown and Willis [4] and others have written about the importance of safety perception surveys. First, as leading indicators of safety performance, they have helped in identifying precursors to accident occurrence, and by so doing, effectively decreased the number of accidents. Second, by providing proactive information about safety problems before they develop into accidents and injuries, safety perception surveys have provided management with guidance on developing specific safety programmes. Third, compared to other proactive means of accident prevention, safety perception analyses are relatively inexpensive. Finally, they have provided information about safety management from employees' perspectives. With specific reference to the current study, exploring the impact of job experience in a safety climate analysis could provide a potent proactive safety management tool, as they could indicate a need for special safety programmes for a particular group.

Given the critical importance of safety climate and the evidence that it is influenced by organizational and human factors, the extent to which safety perceptions differ in different work groups, companies and institutions has been

Correspondence and requests for offprints should be sent to Seth Ayim Gyekye, Psychology Department, Buckinghamshire New University, Queen Alexandra Rd, High Wycombe, Bucks, HP11 2JZ, UK. E-mail: <gas.gyekye@bucks.ac.uk>. 
meticulously examined for the past 30 years. DeJoy, Murphy and Gershon's analyses in health care settings [5], Diaz and Cabrera's analyses on airport ground handling operations [6] and Niskanen's study on road administration [7] are examples of such studies. Additionally, comparative analyses between managers' and employees' perceptions [4], high- and lowaccident organizations [3], individual- and organizational-level climate perceptions [8, 9] and between blue- and white-collar workers [10] have been carried out. What most of these studies revealed is that workers differ in their attitudes to safety issues and in their perceptions of workplace hazards. A body of evidence also links safety perception to safety performance [2, 8], accident frequency [11], job satisfaction [12], compliance with safety management polices $[13,14]$ and workers' perceptions regarding organizational support $[15,16]$.

Several publications on workplace injury and safety management point to the increasing importance of workers' demographic variables, particularly job experience (e.g., Carder and Ragan [17], Hansen [18], Fabiano, Currò, Reverberi, et al. [19] and Paul and Maiti [20]). Despite this attention, there is still paucity of research regarding the impact of workers' experience on workplace safety perceptions. The current study was thus designed to extend the study on work experience and safety management. Accordingly, it compared the safety perceptions of workers with two levels of job experience (experienced/inexperienced). Additionally, it investigated the relationship between these experience levels with job satisfaction, compliance with safety management procedures, and accident frequency. The dearth in research on organizational behaviour in the African work environment constitutes another reason for these analyses.

\section{Work Experience in the Accident and Safety Literature}

Workers' experience is one of the most extensively researched concepts in the organizational literature. It is regarded as one of the most relevant job characteristics for predicting job performance, but with some conceptual disagreement. It is sometimes defined in the organizational literature as the job-relevant knowledge gained over time (e.g., Quiñones, Ford and Teachout [21]), and at times as organizational tenure $[22,23]$. A considerable body of literature has linked work experience to a range of organizational performances including risk-taking, accident analysis and safety management. Most studies in this domain have focused predominantly on experienceaccident frequency relationships, and research specifically designed to explore the relationship between work experience and safety perceptions is lacking.

Research studies designed to investigate the link between work experience and job satisfaction is scanty. Much of the theoretical and empirical research has investigated the association between organizational tenure and job satisfaction, and the conclusions drawn have been deduced from the affective organizational attachment that develops with tenure (e.g., Ambrose and Cropanzano [24] and Wright and Bonett [25]). Through this link, work experience has been found to be a strong predictor of job satisfaction (e.g., Trevor [26] and Carsten and Spector [27]). The argument for this observation is that longevity at work builds up a psychological link between workers and their organizations through which longtenured workers (experienced) identify with their organizations and experience more positive feelings about their job assignments and work conditions. Consequently, relative to their shorttenured colleagues (inexperienced), long-tenured (experienced) workers expressed greater job satisfaction. This relationship has been confirmed and highlighted in a review by Oshagbemi [28].

However, other researchers have found that longevity at the workplace does not necessarily bring loyalty, commitment and indicate job satisfaction (e.g., Wright and Bonett [25] and Kass, Vodanovich and Callender [29]). Kass et al. found a positive association between job dissatisfaction, boredom, and organizational tenure; they argued that longevity was due to workers being tied to their workplaces and 
lacking suitable job alternatives [29]. In essence, longevity cannot be regarded as a consistent indicator of organizational attachment and job satisfaction. There is therefore no obvious basis to expect long-tenured (experienced) workers to express greater job satisfaction than their shorttenured (inexperienced) counterparts.

Studies on the link between work experience and compliance with safety policies is rather inadequate and conflicting. While Zeitlin [30] found overall safety compliance to be highest among the inexperienced, Paul and Maiti [20] in their recent study, did not find any such relationship. By means of multivariate methodology, they recorded that the experienced, as well as their inexperienced counterparts, are likely to disregard safety policies. Research on work experience and accident frequency is rather consistent with a few contradictory findings. While a substantial body of research found that length of job experience was related to decreased accident rates (e.g., Fabiano et al. [19], Barkan, Zohar and Erev [31], Kecojevic, Komljenovic, Groves, et al. [32], Wu, Liu and Lu [33], Siu, Phillips and Leung [34]), a few studies found experience to be positively related to accident frequency (e.g., Hansen [18] and Frone [35]). The principal reason for this observation was the close association between experience and job requirements which entail greater skill requirements, responsibility and exposure to greater accident risk. Yet, other studies have shown that experience per se does not decrease accident frequency independently of age [20, 36]. In an empirical study of three groups of various experience working in the same area and exposed to the same environmental hazards, the relationship between accident frequency and work experience was found to follow the shape of an inverted $U$ [37]. Workers with the least experience (probationary: 1-3 months' experience), and workers with the most experience (incumbents: 12 months' and over) had significantly fewer accidents than workers with intermediate experience (recent hires: 3-12 months' experience). Butani recorded injuries among coal miners to vary more by experience than by age [38]. He noted that workers were experiencing injuries at the same rate when grouped by age, but not when grouped by experience. According to Paul and Maiti an inexperienced worker is equally likely to be injured as an experienced one [20].

On the basis of this literature review, it is reasonable to expect employees' work experience to have different effects on their safety perceptions, level of job satisfaction, compliance with safe work procedures and accident frequency. Thus the following hypotheses were formulated.

- Hypothesis 1: work experience and safety perception. Because of the absence of ample evidence that bears directly on this relationship, it is tested but no prediction is made regarding its direction.

- Hypothesis 2: work experience and job satisfaction. Despite the irregularities and inconsistencies, it is anticipated that experienced workers would express more job satisfaction than their inexperienced colleagues.

- Hypothesis 3: work experience and compliance with safety work procedures. Because of the absence of ample evidence that bears directly on this relationship and the inconsistencies in the few findings, this relationship is tested but no prediction is made regarding its direction.

- Hypothesis 4: work experience and accident frequency. Despite the irregularities and inconsistencies, it is anticipated that experienced workers would have fewer accidents.

\section{METHOD}

\subsection{Sample and Procedure}

Three hundred and twenty Ghanaian industrial workers participated in the study; $32 \%(n=102)$ of them were miners, while 68\% $(n=218)$ were from textiles, timber and saw-mill plants, breweries, and food-processing plants in the industrial cities of Accra, Tema and Kumasi. Sixty-five percent $(n=208)$ were male and $35 \%$ $(n=112)$ female. Thirteen percent $(n=42)$ of all participants had been at the workplace for 
less than a year; $22 \%(n=70) 1-5$ years; $21 \%$ $(n=67) 5-11$ years; $25 \%(n=80) \quad 11-15$ years; and $19 \%(n=61)$ over 15 years. Regarding age, $22 \%(n=70)$ of the workers were 18-29 years old; $25 \%(n=80)$ 30-39; 43\% ( $n=134) 40-49$, and $10 \%(n=32)$ were 50 years old and more. The educational background of the participants was as follows: 50\% $(n=159)$ had basic education, $30 \%(n=98)$ had secondary education, 17\% $(n=56)$ had professional education and $3 \%(n=7)$ had university education.

During lunch break, participants responded to a structured questionnaire in English, which took 15-20 min to complete. Supervisors completed the questionnaire unaided, while for illiterate or semiliterature respondents who had difficulty understanding written English, the local language was used via an interpreter. Respondents were assured that their responses would remain anonymous and confidential and that none of their managers were involved in any way in the study. Statistical analyses of the data were carried out with SAS version 8.2.

\subsection{Questionnaire}

2.2.1. Perceptions of safety climate were measured with the 50-item work safety scale developed by Hayes, Perander and Smecko [39]. Participants responded on a 5-point scale ranging from 1-not at all to 5-very much. This instrument assesses employees' perceptions on work safety and measures 5 distinct constructs, each with 10 items: (a) work safety (sample item: safety programmes are effective, $\alpha=.96$ ), (b) co-workers' safety (sample item: pay attention to safety rules, $\alpha=.80$ ), (c) supervisor safety (sample item: enforces safety rules, $\alpha=.97$ ), (d) management's commitment to safety (sample item: responds to safety concern, $\alpha=.94$ ), (e) satisfaction with safety programme (sample item: effective in reducing accidents, $\alpha=.86$ ).

2.2.2. Work experience was measured in terms of organizational tenure (e.g., Cellier, Eyrolle and Bertrand [22] and Savery and Wooden [23]). It was thus measured with participants' responses to the question: how long have you worked in this organization? Response options were: (a) 1-12 months, (b) 1-5 years, (c) 5-11 years, (d) 11-15 years, (e) over 15 years. Following previous studies, workers with 1-5 years' tenure were categorized as inexperienced, and those with 5 years and more as experienced.

2.2.3. Age and job role. Respondents indicated their age and job status (supervisor/subordinate) by marking the appropriate choice in response to a dichotomous item on the questionnaire.

\subsubsection{Job satisfaction represented workers'} overall feelings towards their jobs. It was measured with one-item global measure of job satisfaction [40]. This measure was chosen because single-item measures of overall job satisfaction have been considered to be equally robust and valid as multiple-item measures (e.g., Nagy [41] and Wanous, Reichers and Hudy [42]). Besides, it has been used extensively in the organizational behaviour literature (e.g., Gyekye [12], Poon [43], Harter, Hayes and Schmidt [44]). The measure has five response categories ranging from extremely dissatisfied to extremely satisfied, corresponding to our 5-point response format 1—not at all to 5-very much.

\subsubsection{Items for compliance with safe work} procedures were pooled from the literature, and comprised of the following four: Follow safety procedures regardless of the situation, Encourage co-workers to be safe, Use appropriate tools and equipment, Follow the correct procedure. Participants responded on a 5-point scale ranging from 1 - not at all to 5-very much $(\alpha=.80)$.

2.2.6. Accident frequency was measured with participants' responses to question in which they were asked to indicate the number of times they had been involved in accidents in the past 12 months. Cases studied were accidents safety inspection authorities classified as serious.

\section{RESULTS}

Four statistical techniques were employed to explore the relationships between the variables. First, a $\chi^{2}$ test showed a highly significant relationship between work experience and 
education $\left(\chi^{2}=243.57, d f=12, p<.001\right)$. As reflected in Table 1, the higher the workers' educational attainment, the more experienced they were.

Second, ANOVA computations indicated differences of statistical significance between the two cohorts of experienced and inexperienced workers. Results of the eight dependent variables with work experience groups revealed significant differences for all dependent variables: work safety $(F(4,296)=90.55, p<.001)$, co-workers safety $(F(4,290)=63.48, p<.001)$, supervisor commitment $(F(4,302)=92.39, p<.001)$, management's commitment $(F(4,300)=45.25$, $p<.001)$, satisfaction with safety programmes $(F(4,255)=83.02, p<.001)$, job satisfaction $(F(4,304)=43.73, p<.001)$, compliance with safety work procedures $(F(4,296)=78.21$, $p<.001)$, and accident frequency $(F(4,299)=79.22, p<.001)$.
In regard to perceptions of work safety, workers with over 15 years' experience perceived their job assignments to be safe (Table 2). Their counterparts with less than a year's experience were the least enthusiastic about the safety levels of their job roles. Workers with over 15 years' experience rated their co-workers' contributions to workplace safety highest. Their inexperienced counterparts (1-12 months) expressed negativity. Again, experienced workers (15+ years) indicated more concern for safety than their supervisors; whereas, their inexperienced counterparts (1-12 months) indicated the least. Experienced workers (11-15; over 15 years) had positive and constructive views concerning management's commitment to safety, and were appreciably enthusiastic about the effectiveness of the safety programmes in place. Meanwhile, their inexperienced counterparts (1-12 months)

TABLE 1. Work Experience of Ghanaian Industrial Workers by Education $\left(x^{2}=243.57, d f=12\right.$, $p<.001)$

\begin{tabular}{|c|c|c|c|c|}
\hline \multirow[b]{2}{*}{ Work Experience } & \multicolumn{4}{|c|}{ Educational Level (\%) } \\
\hline & Basic $(n=159)$ & Secondary $(n=98)$ & Professional $(n=56)$ & University $(n=7)$ \\
\hline $1-12$ months & 51 & 1 & 2 & 0 \\
\hline $1-5$ years & 34 & 34 & 3 & 25 \\
\hline 5-11 years & 6 & 41 & 11 & 25 \\
\hline $11-15$ years & 4 & 18 & 44 & 25 \\
\hline$>15$ years & 5 & 6 & 40 & 25 \\
\hline total & 100 & 100 & 100 & 100 \\
\hline
\end{tabular}

TABLE 2. Descriptive Statistics on Workplace Safety Scale and Work Experience

\begin{tabular}{|c|c|c|c|c|c|c|c|c|c|c|c|}
\hline \multirow[b]{3}{*}{ Work Safety } & \multicolumn{4}{|c|}{ Inexperienced Workers } & \multicolumn{6}{|c|}{ Experienced Workers } & \multirow[b]{3}{*}{$P$} \\
\hline & \multicolumn{2}{|c|}{ 1-12 months } & \multicolumn{2}{|c|}{$1-5$ years } & \multicolumn{2}{|c|}{ 5-11 years } & \multicolumn{2}{|c|}{$11-15$ years } & \multicolumn{2}{|c|}{$>15$ years } & \\
\hline & $M$ & $S D$ & $M$ & $S D$ & $M$ & $S D$ & $M$ & $S D$ & $M$ & $S D$ & \\
\hline Dangerous & 4.00 & 1.32 & 3.65 & 1.46 & 2.37 & 1.27 & 1.96 & 1.28 & 1.57 & 1.23 & $\star \star \star *$ \\
\hline Safe & 1.87 & 1.43 & 2.14 & 1.43 & 3.18 & 1.25 & 3.60 & 1.24 & 4.22 & 1.16 & *** \\
\hline Hazardous & 3.32 & 1.63 & 3.34 & 1.41 & 2.43 & 1.28 & 1.60 & 1.01 & 1.49 & 1.01 & *** \\
\hline Risky & 3.66 & 1.55 & 3.78 & 1.25 & 2.06 & 1.18 & 1.75 & 0.82 & 1.36 & 0.75 & $\star \star \star \star ~$ \\
\hline Unhealthy & 3.86 & 1.36 & 3.72 & 1.23 & 2.12 & 0.96 & 1.59 & 0.72 & 1.49 & 0.85 & *** \\
\hline Could get hurt & 3.84 & 1.36 & 3.76 & 1.17 & 2.08 & 1.13 & 1.59 & 1.03 & 1.54 & 0.82 & *** \\
\hline Unsafe & 4.11 & 1.01 & 3.91 & 1.21 & 2.25 & 1.13 & 1.65 & 0.83 & 1.60 & 0.81 & *** \\
\hline Fear of death & 3.84 & 1.35 & 3.81 & 1.20 & 2.26 & 1.19 & 1.58 & 0.77 & 1.49 & 0.85 & $\star \star * *$ \\
\hline Chance of death & 3.73 & 1.48 & 3.56 & 1.30 & 2.07 & 1.13 & 1.40 & 0.71 & 1.43 & 0.85 & *** \\
\hline Scary & 3.79 & 1.44 & 3.65 & 1.13 & 2.06 & 1.17 & 1.64 & 0.82 & 1.34 & 0.70 & *** \\
\hline
\end{tabular}

Notes. ${ }^{* *} p<.001$. Scores on work safety were in reverse order. 
TABLE 2. (continued).

\begin{tabular}{|c|c|c|c|c|c|c|c|c|c|c|c|}
\hline \multirow[b]{3}{*}{ Co-Worker Safety } & \multicolumn{4}{|c|}{ Inexperienced Workers } & \multicolumn{6}{|c|}{ Experienced Workers } & \multirow[b]{3}{*}{$\boldsymbol{P}$} \\
\hline & \multicolumn{2}{|c|}{ 1-12 months } & \multicolumn{2}{|c|}{$1-5$ years } & \multicolumn{2}{|c|}{ 5-11 years } & \multicolumn{2}{|c|}{ 11-15 years } & \multicolumn{2}{|c|}{$>15$ years } & \\
\hline & $M$ & $S D$ & $M$ & $S D$ & $M$ & $S D$ & $M$ & $S D$ & $M$ & $S D$ & \\
\hline Ignores safety rules & 3.44 & 1.22 & 3.12 & 1.23 & 2.45 & 0.93 & 1.98 & 0.95 & 1.70 & 0.94 & $* \star *$ \\
\hline $\begin{array}{l}\text { Doesn't care about others' } \\
\text { safety }\end{array}$ & 3.34 & 1.49 & 3.21 & 1.22 & 2.25 & 1.05 & 2.01 & 1.11 & 1.58 & 1.01 & *** \\
\hline Pays attention to safety rules & 2.28 & 1.14 & 2.51 & 1.27 & 3.22 & 1.05 & 3.79 & 0.87 & 3.93 & 0.99 & $* \star *$ \\
\hline Follows safety rules & 1.11 & 1.20 & 2.50 & 1.15 & 3.25 & 1.08 & 4.25 & 0.80 & 4.45 & 0.89 & $\star * *$ \\
\hline Looks out for other's safety & 2.05 & 1.18 & 2,56 & 1.32 & 3.77 & 1.15 & 4.40 & 0.73 & 4.44 & 0.79 & $\star \star \star$ \\
\hline Encourages others to safety & 2.00 & 1.01 & 2.41 & 1.08 & 3.30 & 0.89 & 3.83 & 0.76 & 4.18 & 0.89 & $\star \star \star *$ \\
\hline Takes chances with safety & 3.42 & 0.73 & 2.34 & 1.09 & 2.88 & 1.04 & 3.41 & 1.12 & 1.82 & 1.32 & $\star \star \star *$ \\
\hline Keeps work area clean & 2.00 & 0.96 & $1 / 36$ & 1.11 & 3.20 & 0.98 & 3.86 & 0.75 & 4.10 & 0.78 & 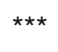 \\
\hline Safety oriented & 1.89 & 1.24 & 2.37 & 1.16 & 3.55 & 1.14 & 4.20 & 0.89 & 4.38 & 0.01 & $* * *$ \\
\hline Doesn't pay attention & 2.18 & 0.89 & 2.57 & 0.99 & 2.71 & 1.16 & 2.38 & 1.17 & 2.46 & 1.22 & ns \\
\hline
\end{tabular}

\begin{tabular}{|c|c|c|c|c|c|c|c|c|c|c|c|}
\hline \multirow[b]{3}{*}{ Supervisor Safety } & \multicolumn{4}{|c|}{ Inexperienced Workers } & \multicolumn{6}{|c|}{ Experienced Workers } & \multirow[b]{3}{*}{$\boldsymbol{P}$} \\
\hline & \multicolumn{2}{|c|}{ 1-12 months } & \multicolumn{2}{|c|}{$1-5$ years } & \multicolumn{2}{|c|}{ 5-11 years } & \multicolumn{2}{|c|}{$11-15$ years } & \multicolumn{2}{|c|}{$>15$ years } & \\
\hline & $M$ & $S D$ & $M$ & $S D$ & $M$ & $S D$ & $M$ & $S D$ & $M$ & $S D$ & \\
\hline Praises safe work behaviour & r 2.56 & 0.79 & 2.64 & 0.88 & 3.44 & 0.77 & 3.68 & 0.63 & 4.25 & 0.69 & 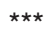 \\
\hline $\begin{array}{l}\text { Encourages safe work } \\
\text { behaviour }\end{array}$ & 2.20 & 0.80 & 2.31 & 1.04 & 3.48 & 1.05 & 3.92 & 0.70 & 4.21 & 0.98 & *** \\
\hline $\begin{array}{l}\text { Informs workers about } \\
\text { safety rules }\end{array}$ & 1.87 & 0.97 & 2.38 & 0.99 & 3.21 & 0.97 & 3.93 & 0.88 & 4.22 & 0.92 & *** \\
\hline Rewards safe behaviours & 1.85 & 0.96 & 2.03 & 1.14 & 2.90 & 1.03 & 3.71 & 1.01 & 3.95 & 1.00 & *** \\
\hline $\begin{array}{l}\text { Involves workers in safety } \\
\text { goals }\end{array}$ & 1.86 & 1.01 & 2.16 & 1.20 & 2.95 & 1.11 & 3.89 & 0.89 & 4.13 & 0.96 & *** \\
\hline Discusses safety issues & 2.10 & 1.11 & 2.16 & 1.10 & 3.09 & 1.20 & 3.96 & 0.72 & 4.36 & 0.88 & *** \\
\hline Updates safety rules & 2.07 & 0.98 & 2.29 & 1.03 & 3.29 & 1.24 & 4.03 & 0.89 & 4.36 & 0.91 & $* * \star$ \\
\hline Trains workers to be safety & 1.84 & 1.01 & 2.34 & 1.14 & 3.47 & 1.26 & 4.07 & 0.75 & 4.39 & 0.88 & *** \\
\hline Enforces safety rules & 1.97 & 0.98 & 2.26 & 1.18 & 3.35 & 1.22 & 4.32 & 0.79 & 4.49 & 0.91 & *** \\
\hline Acts on safety suggestions & 2.18 & 1.14 & 2.32 & 1.25 & 3.67 & 1.17 & 4.36 & 0.81 & 4.65 & 0.79 & *** \\
\hline
\end{tabular}

\begin{tabular}{|c|c|c|c|c|c|c|c|c|c|c|c|}
\hline \multirow[b]{3}{*}{ Management Safety } & \multicolumn{4}{|c|}{ Inexperienced Workers } & \multicolumn{6}{|c|}{ Experienced Workers } & \multirow[b]{3}{*}{$\boldsymbol{P}$} \\
\hline & \multicolumn{2}{|c|}{ 1-12 months } & \multicolumn{2}{|c|}{$1-5$ years } & \multicolumn{2}{|c|}{ 5-11 years } & \multicolumn{2}{|c|}{$11-15$ years } & \multicolumn{2}{|c|}{$>15$ years } & \\
\hline & $M$ & $S D$ & $M$ & $S D$ & $M$ & $S D$ & $M$ & $S D$ & $M$ & $S D$ & \\
\hline $\begin{array}{l}\text { Provides good safety } \\
\text { programme }\end{array}$ & 2.02 & 1.06 & 2.38 & 0.99 & 3.95 & 0.98 & 3.44 & 0.99 & 3.45 & 0.84 & $* \star *$ \\
\hline Conducts safety inspections & 1.66 & 0.95 & 2.07 & 1.01 & 2.54 & 1.06 & 3.02 & 1.07 & 2.58 & 1.05 & $* \star *$ \\
\hline Investigates safety problems & 1.58 & 0.85 & 1.94 & 0.91 & 2.57 & 0.93 & 3.06 & 1.04 & 2.55 & 1.01 & *** \\
\hline Rewards safe workers & 1.69 & 0.73 & 2.01 & 0.92 & 2.40 & 1.04 & 2.76 & 1.87 & 2.57 & 1.00 & *** \\
\hline Provides safe equipment & 1.71 & 0.85 & 2.02 & 0.98 & 2.79 & 1.08 & 3.28 & 0.98 & 3.33 & 0.84 & *** \\
\hline $\begin{array}{l}\text { Provides safe work } \\
\text { conditions }\end{array}$ & 1.85 & 0.96 & 2.07 & 0.95 & 2.90 & 1.03 & 3.36 & 0.98 & 3.45 & 1.07 & $* \star *$ \\
\hline $\begin{array}{l}\text { Responds to safety } \\
\text { concerns }\end{array}$ & 2.02 & 1.03 & 2.10 & 0.95 & 2.95 & 1.10 & 3.42 & 1.08 & 3.67 & 1.16 & $* \star \star$ \\
\hline $\begin{array}{l}\text { Helps maintain workplace } \\
\text { clean }\end{array}$ & 2.00 & 1.00 & 1.95 & 1.17 & 3.03 & 1.29 & 3.50 & 1.10 & 3.89 & 0.98 & *** \\
\hline Provides safety information & 1.97 & 1.11 & 2.35 & 1.06 & 3.26 & 1.31 & 3.82 & 1.15 & 3.92 & 1.07 & *** \\
\hline $\begin{array}{l}\text { Keeps workers informed } \\
\text { about hazards }\end{array}$ & 1.92 & 1.15 & 2.16 & 1.08 & 3.14 & 1.31 & 3.82 & 1.12 & 4.05 & 1.12 & $* * *$ \\
\hline
\end{tabular}

Notes. ${ }^{* *} p<.001$. Scores on work safety were in reverse order. 
TABLE 2. (continued).

\begin{tabular}{|c|c|c|c|c|c|c|c|c|c|c|c|}
\hline \multirow[b]{3}{*}{ Safety Programmes } & \multicolumn{4}{|c|}{ Inexperienced Workers } & \multicolumn{6}{|c|}{ Experienced Workers } & \multirow[b]{3}{*}{$\boldsymbol{P}$} \\
\hline & \multicolumn{2}{|c|}{ 1-12 months } & \multicolumn{2}{|c|}{$1-5$ years } & \multicolumn{2}{|c|}{ 5-11 years } & \multicolumn{2}{|c|}{$11-15$ years } & \multicolumn{2}{|c|}{$>15$ years } & \\
\hline & $M$ & $S D$ & $M$ & $S D$ & $M$ & $S D$ & $M$ & $S D$ & $M$ & $S D$ & \\
\hline Worthwhile & 2.05 & 1.10 & 2.73 & 1.15 & 3.54 & 1.05 & 4.03 & 0.93 & 4.44 & 0.83 & *** \\
\hline Help prevent accidents & 1.97 & 1.13 & 1.96 & 0.96 & 3.34 & 1.10 & 4.05 & 0.94 & 4.35 & 0.76 & *** \\
\hline Useful & 1.71 & 1.02 & 1.83 & 1.22 & 3.48 & 1.27 & 4.23 & 0.77 & 4.42 & 0.89 & *** \\
\hline Good & 1.79 & 1.12 & 1.78 & 1.18 & 3.41 & 1.30 & 4.23 & 0.85 & 4.44 & 0.93 & *** \\
\hline First rate & 1.74 & 1.04 & 1.87 & 1.10 & 3.18 & 1.09 & 4.01 & 0.94 & 4.23 & 0.98 & *** \\
\hline Unclear & 3.39 & 1.07 & 3.00 & 1.07 & 2.62 & 1.00 & 2.15 & 1.21 & 2.27 & 1.36 & *** \\
\hline Important & 1.87 & 1.03 & 1.90 & 1.15 & 3.39 & 1.16 & 3.74 & 0.98 & 4.13 & 0.94 & *** \\
\hline Effective in reducing injuries & 1.82 & 0.82 & 1.93 & 1.20 & 3.39 & 1.32 & 4.05 & 0.88 & 4.14 & 0.94 & *** \\
\hline Do not apply to workplace & 3.30 & 1.05 & 2.77 & 0.93 & 2.10 & 1.08 & 2.30 & 1.77 & 1.92 & 1.37 & *** \\
\hline Do not work & 2.56 & 1.22 & 2.80 & 0.98 & 2.12 & 1.14 & 2.20 & 1.36 & 1.56 & 1.40 & ns \\
\hline
\end{tabular}

Notes. ${ }^{* \star *} p<.001$. Scores on work safety were in reverse order.

expressed negativity and disapproved of the safety programmes. A similar pattern was observed on the organizational variables. The experienced workers, more than their inexperienced counterparts, expressed more job satisfaction, were more compliant with safety work procedures, and recorded a lower accident involvement rate.

Third, because experience, age, and job role are strongly interrelated, as shown by status-attainment models (e.g., Blau [47]), it was necessary to estimate the effect of work experience in a multivariate analysis (MANOVA), controlling for the effects of job role and age. The interaction model (age $\times$ experience $\times$ job role) was highly significant for work safety $(F(26,258)=18.89, p<.001)$. The main effects of experience $(F(4,258)=30.68, p<.001)$, job role $(F(1,258)=75.98, p<.001)$, and age $(F(3,258)=85.87, p<.001)$, and the interaction effects of job role and experience were all significant $(F(3,258)=4.47, p<.010)$. The interaction model for co-worker safety was also highly significant $(F(26,250)=14.35, p<.001)$. The main effects of experience $(F(4,250)=14.47$, $p<.001)$, age $(F(3,250)=78.85, p<.001)$, and job role $(F(1,250)=60.90, p<.001)$ were significant, too. Other interactions were not significant.

The interaction model for supervisors' contributions towards safety was also highly significant $(F(26,264)=19.53, p<.001)$. The main effects of experience $(F(4,264)=17.36$, $p<.001)$, age $(F(3,264)=100.70, p<.001)$ and job role $(F(1,264)=117.75, p<.001)$ were significant. No interaction was significant. Management safety practices indicated a highly significant effect $(F(26,262)=9.54$, $p<.001)$. Again the main effects of experience $(F(4,262)=13.26, p<.001)$, age $(F(3,262)=44.47, p<.001)$, and job role $(F(1,262)=43.15, p<.001)$ were significant, but none of the interaction effects were. Regarding workers' perceptions on safety programmes, the interaction model was highly significant $(F(25,221)=15.01, p<.001)$, with the main effects of job role $(F(1,221)=41.94$, $p<.001)$, age $(F(3,221)=78.44, p<.001)$, and experience $(F(4,221)=20.38, p<.001)$. Other interactions were not significant.

Regarding workers' compliance with safety management procedures, the interaction model (age $\times$ experience $\times$ job role) was highly significant $(F(25,221)=12.45, p<.001)$, and again the significant main effects were experience $(F(4,221)=16.41, p<.001)$, job role $(F(1,221)=37.94, p<.001)$, and age $(F(3,221)=65.61, p<.001)$. There were no significant interaction effects. The interaction effect for job satisfaction was highly significant $(F(26,262)=12.00, p<.001)$. The main effects of experience $(F(4,262)=62.56, p<.001)$, age $(F(3,262)=2.77, p<.05)$, and job role $(F(1,262)=16.06, p<.001)$ were significant. 
TABLE 3. Descriptive Statistics on Workplace Safety Scale, Work Experience and Organizational Variables $(p<.001)$

\begin{tabular}{|c|c|c|c|c|c|c|c|c|c|c|}
\hline \multirow[b]{3}{*}{ Variables } & \multicolumn{4}{|c|}{ Inexperienced Workers } & \multicolumn{6}{|c|}{ Experienced Workers } \\
\hline & \multicolumn{2}{|c|}{ 1-12 months } & \multicolumn{2}{|c|}{$1-5$ years } & \multicolumn{2}{|c|}{ 5-11 years } & \multicolumn{2}{|c|}{$11-15$ years } & \multicolumn{2}{|c|}{$>15$ years } \\
\hline & $M$ & $S D$ & $M$ & $S D$ & $M$ & $S D$ & $M$ & $S D$ & $M$ & $S D$ \\
\hline WSSA & $36.05_{a}$ & 9.47 & $35.35 a$ & 8.89 & $22.88_{b}$ & 7.56 & $18.39_{b}$ & 4.91 & $17.54_{b}$ & 5.52 \\
\hline WSSB & $23.13_{a}$ & 4.94 & $26.12_{a}$ & 5.10 & $30.80_{b}$ & 5.04 & $34.17_{b}$ & 3.45 & $34.85_{b}$ & 4.35 \\
\hline WSSC & $20.52 a$ & 7.81 & $22.94 a$ & 9.19 & $33.03_{b}$ & 9.24 & $39.83_{b}$ & 5.39 & $43.03_{b}$ & 6.93 \\
\hline WSSD & $18.46_{a}$ & 7.63 & $21.00_{a}$ & 7.93 & $28.57_{b}$ & 8.46 & $33.48_{b}$ & 7.68 & $33.49_{b}$ & 6.89 \\
\hline WSSE & $19.11_{\mathrm{a}}$ & 6.36 & $19.96 \mathrm{a}$ & 7.61 & $30.50_{b}$ & 8.00 & $37.39_{b}$ & 6.43 & $38.13_{b}$ & 6.25 \\
\hline Compliance & $10.45_{a}$ & 4.33 & $12.35 a$ & 4.63 & $17.06_{b}$ & 4.03 & $20.13_{b}$ & 2.91 & $21.17_{b}$ & 3.61 \\
\hline Job satisfaction & $1.92 a$ & 1.38 & $2.29_{a}$ & 1.26 & $3.56_{b}$ & 0.94 & $3.88_{b}$ & 0.99 & $4.25_{b}$ & 1.16 \\
\hline Accident frequency & $3.15_{a}$ & 0.97 & $2.73_{a}$ & 0.89 & $1.64_{b}$ & 0.98 & $1.17_{b}$ & 0.44 & $1.10_{b}$ & 0.45 \\
\hline
\end{tabular}

Notes. WSSA—work safety, WSSB—co-worker safety, WSSC—supervisor safety, WSSD—management attitudes and practices, WSSE-safety programmes; scores on work safety were in reverse order. Means with different subscripts in the same row are significantly different at $p<.001$.

The interaction effect of experience and age was also significant $(F(9,262)=2.35, p<.05)$, but not the other interaction effects. The interaction model was highly significant for accident frequency $(F(25,258)=15.70, p<.001)$. The main effects of experience $(F(4,258)=89.97$, $p<.001)$ and age $(F(3,258)=4.04, p<.01)$ were significant, but none of the interaction effects. All in all, the effect of workers' experience was notably high on all these variables.

Fourth, a posterior $t$ test consistently revealed significant differences between the experienced and inexperienced cohorts (Table 3). The experienced workers, compared to their inexperienced counterparts, evaluated their workplace as safer $(t=18.52, d f=299, p<.001)$, saw their co-workers $(t=-15.23, d f=289, p<.001)$, their supervisors $(t=-16.31, d f=305, p<.001)$, and management as more committed to safety $(t=-12.35, d f=303, p<.001)$, were more satisfied with the safety programme $(t=-16.01$, $d f=258, p<.001)$, and with their jobs $(t=-12.47$, $d f=303, p<.001)$, were more compliant with safety work procedures $(t=-15.28, d f=258$, $p<.001)$, and had experienced fewer accidents in the past 12 months $(t=16.43, d f=298, p<.001)$.

\section{DISCUSSION}

This current study investigated the impact of work experience on workplace safety perceptions by comparing two groups divided according to their experience. It also examined the relationships between work experience, job satisfaction, compliance with safety management policies, and accident frequency. The major finding was an association between workers' level of experience and perception of workplace safety. The more experienced workers had more constructive perspectives regarding safety than their inexperienced counterparts. Additionally, they also expressed the highest level of job satisfaction, complied with safety policies, and recorded the lowest accident involvement rate. Inexperienced workers, particularly those in their first year at the workplace, were the worst perpetrators regarding safety perception, job satisfaction, compliance with safety policies, and accident involvement rate.

The observed positive relationship between experience and safety perception could be explained by the concept of familiarity and perception of hazards. Apparently, the more experienced workers (particularly those with over 15 years of experience) benefited from their longevity with their organizations. They had acquired the specific job-related and organizational relevant knowledge and the awareness of the organization's safety culture. 
Additionally, they had gained seniority and moved to safer jobs that rarely exposed them to the risks and hazards that their relatively inexperienced counterparts encountered. In many workplaces, it is not uncommon to find long-tenured and experienced workers act simultaneously as superiors and subordinates in the workplace. Quite often, they deputize for supervisors and assign to themselves the less risky and dangerous jobs, while leaving the more risky and less desirable ones to the new and inexperienced workers.

As anticipated, the finding supported a positive association between work experience and job satisfaction. Workers with 15 years' experience were the most satisfied with workplace conditions. As tenure increased, the workers found a complex job less novel and interesting. These assignments were most often mentally challenging with greater discretion. Field work experience and interactions revealed that they had access to the bureaucratic managerial system, and had more resources at their disposal than their relatively new and inexperienced counterparts. Their perceptions regarding these positive effects and favours from their organizations created a feeling of indebtedness and a corresponding sense of obligation for them to respond positively in return. As a means of reciprocity ${ }^{1}$ for the management's recompense, they complied with the safety management policies [16, 45], and subsequently recorded a relatively lower rate of injury involvement.

The observed negative relationship between work experience and accident frequency could be further explained by the strong association between job experience and organizational tenure (e.g., Cellier et al. [22] and Hale and Glendon [46]). According to these findings, awareness of safety, sensitivity to hazardous situations, diligence and assiduousness tend to increase with tenure. The higher longevity at work provided experienced workers with skills, greater organizational knowledge of safe working procedures, which enhanced their job performance. Hence, they learned to handle successfully unexpected situations, and displayed acumen and prudence in their ability to recognise situational contingencies, carefully appraised them and avoided disaster. Our observations are consistent with previous findings: both older [38] and more recent ones [19, 32, 33].

Furthermore, experienced workers were most often considered as permanent staff and received safety-related training that provided them with greater knowledge and skills regarding appropriate safety behaviours (e.g., compliance with safety policies). As protagonists and enforcers of safety rules, experienced workers were motivated to comply with the organization's safe work procedures to manifest themselves as responsible role models $[9,49,50]$. Workers who possess the requisite knowledge in the very specific organizational context within which hazards are encountered (safety orientation) tend to be more compliant with safety policies (e.g., Probst [13], Probst and Brubaker [14] and Zeitlin [30]).

By contrast, inexperienced workers, particularly those with less than a year's experience, with comparatively less normative knowledge, and less familiarity with the workplace conditions, were at a greater risk of displaying inappropriate and inaccurate safety behaviours. Thus, they misunderstood and the violated more organizational safety policies, and hence recorded the highest accident involvement rate. It is not uncommon to also find this category of workers accepting hazardous tasks and working under considerable stressful conditions, such as job and income insecurity, low earnings, and inability to raise workplace issues and concerns: issues which presumably lead to negative perceptions regarding workplace safety, low job satisfaction, and increased vulnerability to accidents.

\footnotetext{
1 According to the social exchange [47] and reciprocity theory [48], workers who perceive some form of organizational support from the management are motivated to reciprocate in terms that benefit their organizations.
} 


\section{SAFETY IMPLICATIONS AND DIRECTIONS FOR FURTHER STUDIES}

The current findings have implications for workplace safety management. Mandatory safety programmes should be put in place for new recruits. This could consist of an integrated approach of education, enforcement and engineering controls which will best protect workers from accidents and injuries. Effective safety education programmes in conjunction with appropriate leadership role (e.g., corrective leadership and supervisory safety practices) will induce positive safety behaviours into subordinate workers on the shop level. Support for this argument comes from Zohar's recent studies in which supervisory monitoring, prioritizing safety over competing goals, and corrective leadership provided complementary modes of influence on safety behaviour $[9,49$, 50]. Additionally, management could invest in making their organizations more satisfying and safer. They could do this by implementing fairness perception measures [51, 52], creating job enrichment programmes [53], and providing support to workers beyond what is formally stated in the contractual agreement [54, 55]. These measures would address the noted disfavoured perceptions regarding supervisors' and management's role in safety from the inexperienced workers' perspectives. Ultimately, they would increase workers' job-satisfaction, organizational efficiency and productivity, inspire safe work practices, and thereby decrease accident frequency and its associated high human and social costs.

The primary strength of this study is its empirical disposition as participants were authentic workers. The findings complement previous ones that found a negative association between job experience and accident frequency (e.g., Fabiano et al. [19], Kecojevic, et al. [32], Siu et al. [34], Frone [35], and Salminen $[56,57])$, and positive association between experience and job satisfaction (e.g., Oshagbemi [28]). It also supports studies that showed that workers with the requisite knowledge of safe work behaviours tend to comply with their organizations' safety management policies and subsequently record fewer accidents (e.g., Elangovan, Mohammed and Mohan [58] and Neal, Griffin and Hart [59]). More importantly, it makes novel contributions to the safety literature on the relationship between work experience and safety perceptions, and compliance with safety management policies.

It is, however, limited by its reliance on self-reported instruments. The possibility thus exists for the findings to be distorted by the participants' desire to respond in a consistent manner. However, recent Crampton and Wagner's meta-analytic research indicated that while this problem continued to be regularly cited, the magnitude of distortions could be overestimated [60]. Self-reported measures have been effectively used in workplace accident analyses and safety surveys (e.g., Gyekye and Salminen [15, 61] and Siu et al. [34]). Besides, while epidemiologic reports were found to be faulty, biased and deficient because of poor documentation [62, 63], research reports have found self-reported accident rates to be closely related to documented accident rates [64]. Notwithstanding these limitations, the current study contributes to the growing body of research, which has identified work experience as an important variable for investigation in safety management policies.

\section{REFERENCES}

1. Zohar D. Safety climate in industrial organizations: theoretical and applied implications. J Appl Psychol. 1980;65: 96-102.

2. Cooper MD, Phillips RA. Exploratory analysis of the safety climate and safety behavior relationship. J Safety Res. 2004; 35:497-512.

3. Zohar D. A group-level model of safety climate: testing the effect of group climate on microaccidents in manufacturing jobs. $\mathrm{J}$ Appl Psychol. 2000:85:587-96.

4. Prussia GE, Brown KA, Willis GP. Mental models of safety: do managers 
and employees see eye to eye? J Safe Res. 2003;34:143-56.

5. DeJoy DM, Murphy LR, Gershon RRM. Safety climate in health care settings. In: Bitent AC, Champney PC, editors. Advances in industrial ergonomics and safety VII. New York, NY, USA: Taylor \& Francis; 1995. p. 923-9.

6. Isla Díaz R, Cabrera D. Safety climate and attitude as evaluation measures of organizational safety. Accid Anal Prev. 1997;29:643-50.

7 Niskanen T. Safety climate in the road administration. Saf Sci. 1994;17:237-55.

8. Hofmann DA, Stetzer A. A cross-level investigation of factors influencing unsafe behaviours and accidents. Personnel Psychology. 1996;49:307-39.

9. Zohar D, Luria G. The use of supervisory practices as leverage to improve safety behavior: a cross-level intervention model. J Safe Res. 2003;34:567-77.

10. Morris WR, Conrad KM, Marcantonio RJ, Marks BA, Ribisl KM. Do blue-collar workers perceive the worksite health climate differently than white-collar workers? Am J Health Promot. 1999;13: 319-24.

11. Guastello SJ, Gershon RM, Murphy LR. Catastrophe model for the exposure to blood-borne pathogens and other accidents in health care settings. Accid Anal Prev. 1999;31:739-49.

12. Gyekye SA. Workers' perceptions of workplace safety and job satisfaction. International Journal of Occupational Safety and Ergonomics (JOSE). 2005;11:291-302.

13. Probst TM. Layoffs and tradeoffs: production, quality, and safety demands under the threat of a job loss. J Occup Health Psychol. 2002;7:211-20.

14 Probst TM, Brubaker TL. The effects of job insecurity on employee safety outcomes: cross-sectional and longitudinal explorations. J Occup Health Psychol. 2001;6:139-59.

15. Gyekye SA, Salminen S. Workplace safety perceptions and perceived organizational support: do supportive perceptions influence safety perceptions? International
Journal of Occupational Safety and Ergonomics (JOSE). 2007;13(2):189-200.

16. Hoffman DA, Morgeson FP. Safety-related behaviour as a social exchange: the role of perceived organizational support and leader-member exchange. J Appl Psychol. 1999;84:286-96.

17. Carder B, Ragan PW. A survey-based system for safety measurement and improvement. J Safety Res. 2003;34(2):157-65.

18. Hansen CP. A causal model of the relationship among accidents, biodata, personality and cognitive factors. J Appl Psychol. 1989;74:81-90.

19. Fabiano B, Currò F, Reverberi AP, Pastorino R. A statistical study on temporary work and occupational accidents: specific risk factors and risk management strategies. Saf Sci. 2008;46(3):535-44.

20. Paul PS, Maiti J. The role of behavioural factors on safety management in underground mines. Saf Sci. 2007;45(4):449-71.

21. Quiñones MA, Ford JK, Teachout MS. The relationship between work experience and job performance: a conceptual and metaanalytic review. Personnel Psychology. 1995;48:887-910.

22. Cellier JM, Eyrolle H, Bertrand A. Effects of age and level of work experience on occurrence of accidents. Percept Mot Skills. 1995;80:931-40.

23. Savery LK, Wooden M. The relative influence of life events and hassles on work-related injuries: some Australian evidence. Hum Relat. 1994;47:283-305.

24. Ambrose ML, Cropanzano R. A longitudinal analysis of organizational fairness: an examination of reactions to tenure and promotion decisions. J Appl Psychol. 2003; 88:266-75.

25. Wright TA, Bonett DG. The moderating effects of employee tenure on the relation between organizational commitment and job performance: a meta-analysis. J Appl Psychol. 2002;87:1183-90.

26. Trevor CO. Interactions among actual ease-of-movement determinants and job satisfaction in the prediction of voluntary turnover. Acad Manage J. 2001;44:621-38.

27. Carsten JM, Spector PE. Unemployment, job satisfaction and employee turnover: a 
meta-analytic test of the Muchinsky model. J Appl Psychol. 1987;72(3):374-81.

28. Oshagbemi T. Personal correlates of job satisfaction: empirical evidence from UK universities. Int J Soc Econ. 2003;30(12): 1210-32.

29. Kass SJ, Vodanovich SJ. Callender A. State-trait boredom: relationship to absenteeism, tenure, and job satisfaction. $\mathrm{J}$ Bus Psychol. 2001;16:317-27 (DOI:10.1023 /A:1011121503118). Retrieved October 29, 2010, from: http://www.springerlink.com/ content/t645m8830hl56411/fulltext.pdf

30. Zeitlin LR. Failure to follow safety instructions: faulty communication or risky decisions. Hum Factors. 1994;36(1):172-81.

31. Barkan R, Zohar D, Erev II. Accidents and decision making under uncertainty: a comparison of four models. Organ Behav Hum Decis Process. 1998;74(2):118-44.

32. Kecojevic V, Komljenovic D, Groves W, Radomsky M. An analysis of equipmentrelated fatal accidents in U.S. mining operations: 1995-2005. Saf Sci. 2007; 45(8):864- 74.

33. Wu TC, Liu CW, Lu MC. Safety climate in university and college laboratories: impact of organizational and individual factors. J Safety Res. 2007;38(1):91-102.

34. Siu OL, Phillips DR, Leung TW. Safety climate and safety performance among construction workers in Hong Kong. The role of psychological strains as mediators. Accid Anal Prev. 2004:36:359-66.

35. Frone MR. Predictors of work injuries among employed adolescents. Appl Psychol. 1998:83(4):565-76.

36. Gun RT, Ryan CF. A case-control study of possible risk factors in the causation of occupational injury. Saf Sci. 1994:18(1):1-13.

37. Keyserling WM. Occupational injuries and work experience. J Safe Res. 1983;14:37-42.

38. Butani SJ. Relative risk analysis of injuries in coal mining by age and experience at present company. Journal of Occupational Accidents. 1988;10:209-16.

39. Hayes BE, Perander J, Smecko T, Trask J. Measuring perceptions of workplace safety: Development and validation of the work safety scale. J Safe Res. 1998:29;145-61.
40. Porter L, Lawler E III. Managerial attitudes and performance. Homewood, IL, USA: Irwin-Dorsey; 1968.

41. Nagy MS. Using a single-item approach to measure job satisfaction. J Occup Organ Psych. 2002:75;77-86.

42. Wanous JP, Reichers AE, Hudy MJ. Overall job satisfaction: how good are single-item measures? J Appl Psychol. 1997:82:247-52.

43. Poon JML. Situational antecedents and outcomes of organizational politics perceptions. Journal of Managerial Psychology. 2003:18(2):138-55.

44. Harter J, Hayes T, Schmidt F. Businessunit-level relationship between employee satisfaction, employee engagement, and business outcomes: a meta-analysis. J Appl Psychol. 2002:87:268-79.

45. Gyekye SA, Salminen S. Are "good soldiers" safety conscious? An examination of the relationship between organizational citizenship behaviors and perceptions of workplace safety. Soc Behav Pers. 2005: 33(8):805-20.

46. Hale A, Glendon A. Individual behaviour in the control of danger. Amsterdam, The Netherlands: Elsevier; 1987.

47. Blau P. Exchange and power in social life. New York, NY, USA: Wiley; 1964.

48. Gouldner AW. The norm of reciprocity: a preliminary statement. Am Sociolol Rev. 1960:25;161-78.

49. Zohar D. Modifying supervisory practices to improve sub-unit safety: a leadershipbased intervention model. J Appl Psychol. 2002;87, 156-63,

50. Zohar D. The effects of leadership dimensions, safety climate, and assigned priorities on minor injuries in work groups. J Organ Behav. 2002;23:75-92.

51. Shore LM, Shore TH. Perceived organizational support and organizational justice. In: Cropanzano RS, Kacmar KM, editors. Organizational politics, justice, and support: managing the social climate of the workplace. Westport, CT, USA: Quorum Books; 1995. p. 149-64.

52. Simons T, Robertson Q. Why managers should care about fairness: the effect of aggregate justice perceptions on organi- 
zational outcomes. J Appl Psychol. 2003: $88 ; 432-43$.

53. Godard J. High performance and the transformation of work:? The implications of alternative work practices for the experience and outcomes of work. Industrial and Labor Relations Review. 2001;54(4):776-805.

54. Aryee S, Budhwar PS, Chen ZX. Trust as a mediator of the relationship between organizational justice and work outcomes: test of a social exchange model. J Organ Behav. 2002:23;267-85.

55. Rhoades L, Eisenberger R. Perceived organizational support: a review of the literature. J Appl Psychol. 2002;87:698-714.

56. Salminen ST. Epidemiological analysis of serious occupational accidents in southern Finland. Scand J Soc Med. 1994:22:225-7.

57. Salminen S. Have young workers more injuries than older ones? An international literature review. J Safety Res. 2004:35; 513-21.

58. Elangovan RK, Mohammed KP, Mohan S. Effectiveness of the designed safety education programme modules by their implementation in selected industries. Journal of Loss Prevention in the Process Industries. 2005:18(4-6):553-7.
59. Neal AF, Griffin MA, Hart PD. The impact of organisational climate on safety climate and individual behaviour. Saf Sci. 2000; 34:99-109.

60. Crampton SM, Wagner JA III. Perceptpercept inflation in microorganizational research: an investigation of prevalence and effect. J Appl Psychol. 1994;79:67-76.

61. Gyekye SA, Salminen S. Educational status and organizational safety climate: does educational attainment influence workers' perceptions of workplace safety? Saf Sci. 2009;47:20-8.

62. Parker DL, Carl WR, French LR, Martin FB. Characteristics of adolescent work injuries reported to Minnesota Department of Labour and Industry. Am J Public Health. 1994;84:606-11.

63. Veazie MA, Landen DD, Bender TR, Amandus HE. Epidemiological research on the etiology of injuries at work. Annu Rev Public Health. 1994:15;203-21.

64. Smith CS, Silverman GS, Heckert TM, Brodke MH, Hayes BE, Silverman MK, et al. A comprehensive method for the assessment of industrial injury events. J Prev Interve Community. 2001:22;5-20. 
